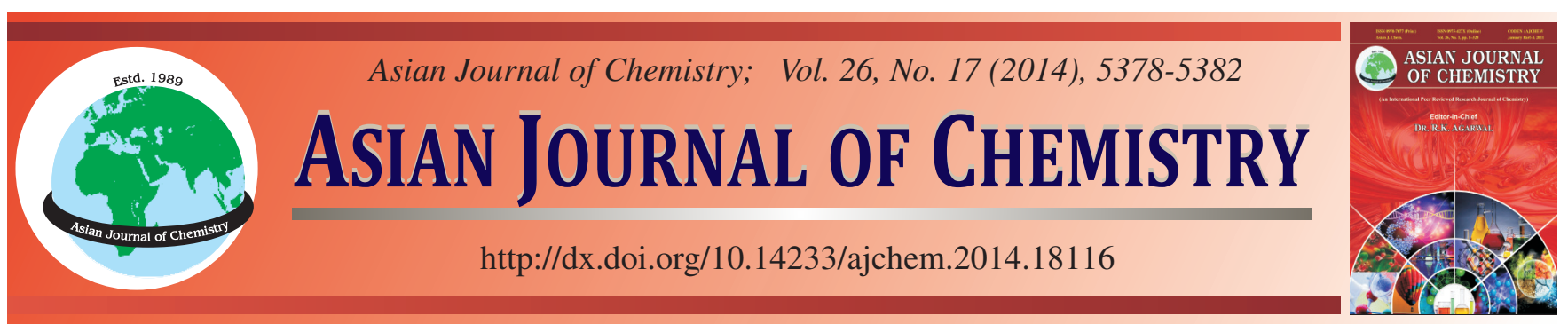

\title{
Molecular Dynamics Simulation of Mechanical Properties for Poly(vinyl pyrrolidone)/Poly(vinyl alcohol) Hydrogel $\dagger$
}

\author{
Y. WANG ${ }^{*}$, X. Li, Q. WeI, W. ChaI, M. YANG and S. WEI
}

Mechanical Engineering School, Northwestern Polytechnical University, Xi'an, P.R. China

*Corresponding author: Fax: +86 29 88491257; Tel: +86 29 88493232; E-mail: wangyanen@ @wpu.edu.cn

Study on the mechanical properties of poly(vinyl pyrrolidone)/poly(vinyl alcohol) (PVP/PVA) composite hydrogel and its interaction mechanism using fully-atomistic molecular dynamics simulation. The mechanical properties and the radial distribution function of three different interpenetration networks including PVP, PVA and (PVP/PVA) have been calculated on material studio platform. Compared with the pure PVP, there is an obvious improvement in the mechanical properties of PVP/PVA and its mechanical properties are not affected by temperature. The interactions between PVA and PVP are expected to occur through interchain hydrogen bonding between the oxygen of PVP and the hydroxyl group of PVA. Molecular dynamics analysis results reveal the interaction mechanism of PVP/PVA hydrogel from microscopic molecular level. Meanwhile, its mechanical properties are stable on the different temperature.

Keywords: Poly(vinyl pyrrolidone), Poly(vinyl alcohol), Molecular dynamics, Mechanical properties.

ᄂ - - - - - - - - - - - - - - - - - - - - - - - - - - - -

\section{INTRODUCTION}

Poly(vinyl pyrrolidone) (PVP), a kind of normal hydrogel for biomedical application, has excellent biological inertness, biocompatibility and good comprehensive performance. However, the PVP hydrogel has relatively low mechanical strength, making its use greatly restricted in the fields which has high requirement of mechanical strength. The mechanical properties of the hydrogel can be modified by processing, such as introducing fibrous reinforcing material, physical blending, grafting reaction and preparation of the interpenetrating network ${ }^{1}$. Poly(vinyl alcohol) (PVA) is well balanced biomedical polymer that has high hydrophilicity and less toxicity on the support of proper mechanical strength. Poly(vinyl pyrrolidone) and poly(vinyl alcohol) organically combined, through mutual penetration or entangled of two kinds of polymer networks, forming interpenetrating polymer network (IPN). It can have excellent properties of general physical blending, grafting and block polymer at the same time, so that the mechanical strength of the hydrogel has been significantly improved.

Many related strategies have focused on composite hydrogel, which afford greater control over each of these aspects, by combining different polymers with tailored chemistries in order to create bioactive systems according to customized functional properties. Additionally, these hydrogels preparation and properties have made great achievements at present, but most of them are from perspective view of experiments and preparation process ${ }^{2-4}$. Few workers carried on the analysis and calculation from the perspective view of microscopic molecular. Therefore, it is particularly necessary to explain the interaction of interfacial mechanism and the mechanical properties of the composite hydrogel material from the microscopic molecular perspective view. In material research fields, molecular dynamics simulation has become an effective and efficient research strategy for investigating the interfacial interactions among the polymer matrixes. It has been employed to explore the mechanical and other chemical reaction mechanism by calculating their binding energies and analyzing the radical distribution and many other properties for the composites materials ${ }^{5,6}$. In this article, based on atomic trajectories calculation theory, molecular dynamics simulation was applied to investigate the mechanical properties and chemical interaction mechanism of these three hydrogels including PVP, PVA and their cross-linked system PVP/PVA. On the basis of the molecular dynamics calculation analysis, their mechanical properties can be demonstrated. The radical distribution will reveal the chemical interfacial interaction among the concrete molecules influence of the cross-linked hydrogels. Therefore, molecular dynamics simulation provides a theoretical approach for composite hydrogel study. 
EXPERIMENTAL

The simulation works are performed with Material Studio platform. In order to getting more accurate simulation results, different force fields were adopted for different material research according to their reaction mechanism during molecular dynamics simulation process.

Due to the purpose of this article is to reveal the biomechanical properties of PVP, PVA and their crosslinked networks PVP/PVA. Therefore, water did not taken into account while building the models. Firstly, according to the molecular structure formula of PVP and PVA, the polymer chains were created with the amorphous module in the Materials Studio. The density of the PVP and PVA was set as 0.98 and $0.96 \mathrm{~g}$ $\mathrm{cm}^{-3}$, respectively, which matches the values of the general commercial products. Three PVP chains with 20 monomers and four PVA chains with 40 monomers were contained in the amorphous cells of PVP and PVA, respectively, as well the atom number of PVP is 1026, the PVA amorphous cell contains 1088 atoms. The number of atoms contained in amorphous cell is close, so that can be compared with each other, as models shown in Fig. 1.

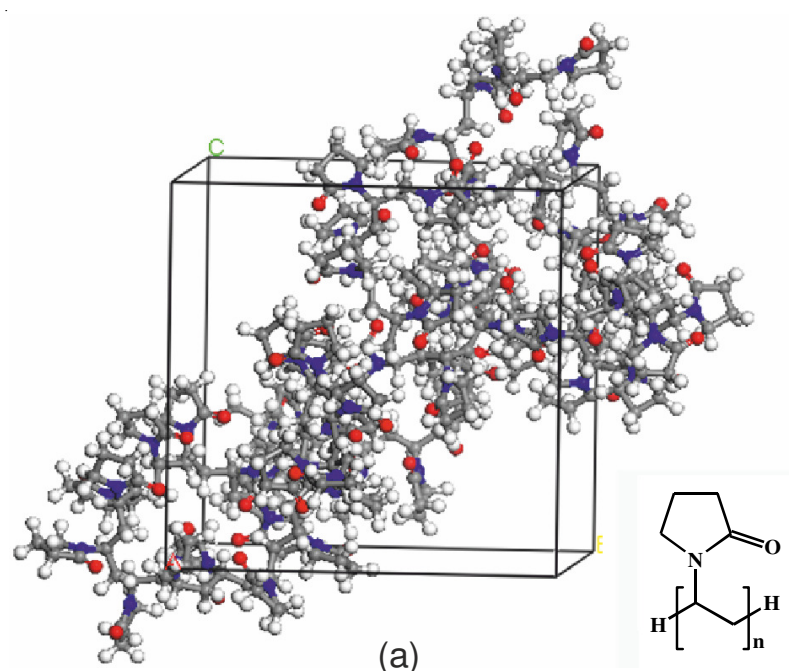

(a)

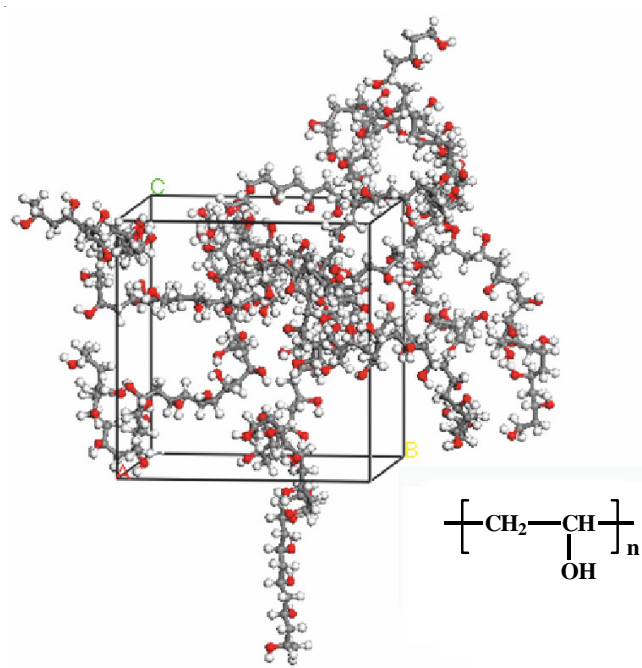

(b)

Fig. 1. Initial geometry structural models before molecular dynamics simulation: (a) represents PVP and (b) represents PVA
Considering the composite hydrogel with PVP as substrate, the PVA as reinforcement and should be compared with a single component, so the mixed model of PVP/PVA contained two chains of 20 monomers PVA and one chain of 40 monomers PVP. The established PVP/PVA hybrid model with atomic number is 966, the model as shown in Fig. 2, where stick-like model is PVA chain; stick-ball model is PVP chain.

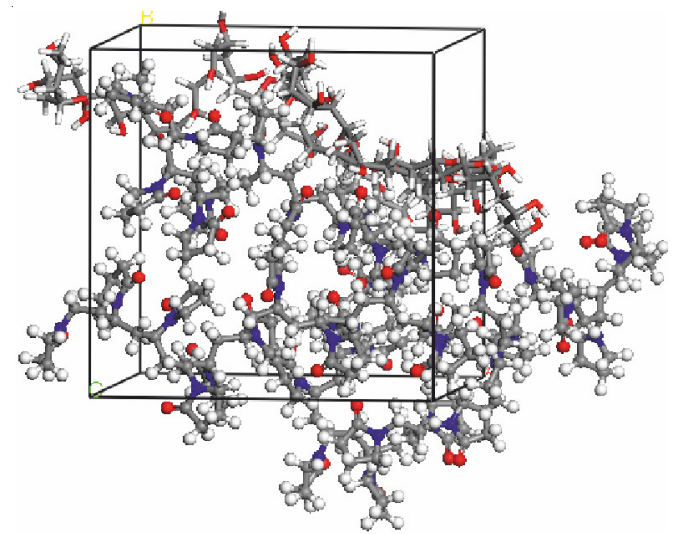

Fig. 2. Initial geometry structure of PVP/PVA before molecular dynamics simulation

Three models were calculated with molecular dynamics simulation under the DISCOVER module of Material Studio. The simulation that was conducted for $40 \mathrm{ps}$ under the canonical ensemble (NVT) and the COMPASS force filed with a time step of $0.1 \mathrm{fs}$ for $30 \mathrm{ps}$ until the system reached equilibrium. Additional 10 ps molecular dynamics calculations were conducted to reveal interaction mechanism of PVP/PVA interpenetration networks. The detailed simulation parameters are shown in Table-1. The mechanical properties can be obtained by analysis of the Elastic Mechanics matrix. Generalized Hooke's law can explore the most materials' general relationship of stress - strain shown as eqn. 1:

$$
\left[\begin{array}{c}
\sigma_{\mathrm{x}} \\
\sigma_{\mathrm{y}} \\
\sigma_{\mathrm{z}} \\
\tau_{\mathrm{yz}} \\
\tau_{\mathrm{zx}} \\
\tau_{\mathrm{xy}}
\end{array}\right]=\left[\begin{array}{llllll}
\mathrm{C}_{11} & \mathrm{C}_{12} & \mathrm{C}_{13} & \mathrm{C}_{14} & \mathrm{C}_{15} & \mathrm{C}_{16} \\
\mathrm{C}_{21} & \mathrm{C}_{22} & \mathrm{C}_{23} & \mathrm{C}_{24} & \mathrm{C}_{25} & \mathrm{C}_{26} \\
\mathrm{C}_{31} & \mathrm{C}_{32} & \mathrm{C}_{33} & \mathrm{C}_{34} & \mathrm{C}_{35} & \mathrm{C}_{36} \\
\mathrm{C}_{41} & \mathrm{C}_{42} & \mathrm{C}_{43} & \mathrm{C}_{44} & \mathrm{C}_{45} & \mathrm{C}_{46} \\
\mathrm{C}_{51} & \mathrm{C}_{52} & \mathrm{C}_{53} & \mathrm{C}_{54} & \mathrm{C}_{55} & \mathrm{C}_{56} \\
\mathrm{C}_{61} & \mathrm{C}_{62} & \mathrm{C}_{63} & \mathrm{C}_{64} & \mathrm{C}_{65} & \mathrm{C}_{66}
\end{array}\right]\left[\begin{array}{c}
\varepsilon_{\mathrm{x}} \\
\varepsilon_{\mathrm{y}} \\
\varepsilon_{\mathrm{z}} \\
\varepsilon_{\mathrm{yz}} \\
\varepsilon_{\mathrm{zx}} \\
\varepsilon_{\mathrm{xy}}
\end{array}\right]
$$

$\mathrm{C}_{\mathrm{ij}}$ is the elastic coefficient matrix elements for $6 \times 6$, a total of 36. In principle, all mechanical properties of materials can be derived from the elastic coefficient matrix, due to the presence of elastic strain energy, that $\mathrm{C}_{\mathrm{ij}}=\mathrm{C}_{\mathrm{ji}}$. Therefore, it describes the stress-strain behaviour of any materials only needs 21 independent elastic. At the atomic level calculation, the stress tensor $\sigma^{7}$ of the static model was calculated by using Virial eqn. 2.

$$
\begin{gathered}
\sigma=-\frac{1}{\mathrm{~V}_{0}}\left[\sum_{\mathrm{i}=1}^{\mathrm{N}} \mathrm{m}_{\mathrm{i}}\left(\mathrm{V}_{\mathrm{i}}, \mathrm{V}_{\mathrm{i}}^{\mathrm{T}}\right)\right] \\
\varepsilon=\frac{1}{2}\left[\left(\mathrm{~h}_{0}^{\mathrm{T}}\right)^{-1} \mathrm{Gh}_{0}^{-1}-1\right]
\end{gathered}
$$


TABLE-1

MOLECULAR DYNAMICS SIMULATION PARAMETERS FOR PVP/PVA

\begin{tabular}{cccccc}
\hline Force field & Non-bond & Summation method & Ensemble & Temperature (K) & Cut-off distance \\
\hline Compass & vdW, Coulomb & Atom based, Ewald & NVT & $260 / 310 / 360 / 410$ & $9.5 \AA$ \\
\hline Thermostat & Barostat & Equilibrium steps & Production steps & Time step & Frame output \\
\hline Andersen & Parrinello & 300,000 & 100,000 & $0.1 \mathrm{fs}$ & Every 200 steps \\
\hline
\end{tabular}

$\mathrm{m}_{\mathrm{i}}$ and $\mathrm{V}_{\mathrm{i}}$ represent the quality and speed of atoms, respectively, $\mathrm{V}_{0}$ is the volume of no distortion. The stress applied to the system makes the relative position of atoms in the system are changed. For a parallelepiped (in simulation, the periodic unit cell' s side lengths are a, b, c ), if the reference state is characterized by the column vectors $\mathrm{a}_{0}, \mathrm{~b}_{0}, \mathrm{c}_{0}$ and the deformation state is characterized by the vector a, b, c, then the strain tensor is eqn. 3 :

In the formula, $\mathrm{h}_{0}$ represents a matrix of column vectors $a_{0}, b_{0}, c_{0} ; h$ is a matrix composed of a, b, c, G represents $h^{T} h$ which is a metric tensor. Therefore, by calculating the slope of the tensile and shear deformation, the elasticity coefficient matrix can be obtained. However, for an isotropic material, there are only two independent elastic coefficients $C_{11}$ and $C_{12}$. For a simple calculation, set $\mathrm{C}_{12}=\lambda, \mathrm{C}_{11}-\mathrm{C}_{12}=2 \lambda, \lambda$ and $\mu$ are Lame coefficients. Thus, modulus of one isotropic material modulus can be expressed by Lame coefficients as following ${ }^{8}$ :

$$
\mathrm{E}=\frac{\mu(3 \lambda+2 \mu)}{\lambda+\mu}, \mathrm{K}=\lambda+\frac{2}{3 \mu}, \mathrm{G}=\mu, \gamma=\frac{\lambda}{2(\lambda+\mu)}
$$

MS program calculated the effective Lame coefficients, elastic modulus, Poisson's ratio and other parameters by assuming the object calculated is isotropic. There are two major criteria to identify whether the molecular dynamics simulation system is equilibrium: one is small fluctuation of temperature and the other is small fluctuation of energy. It requires the both constant or fluctuates slightly up and down along a constant value. In general, the fluctuation of temperature and energy is between 5 and $10 \%$, the system is considered to reach equilibrium. Now, with the PVP/PVA cross-linked system as an example, from the Fig. 3, its temperature and energy curves fluctuate within $5 \%$, which indicate the interpenetration system had reached equilibrium after the molecular dynamics simulation.

Mechanical properties are important basic performance of the relationship between material preparation, processing, production. Table- 2 lists the result based on molecular dynamics trajectory and the static mechanics study results of the above two kinds of the hybrid system and the effective isotropic elastic coefficient of mechanical properties, including tensile modulus E, body modulus $\mathrm{K}$, shear modulus $\mathrm{G}$ and the Cauchy stress $\left(\mathrm{C}_{12}-\mathrm{C}_{44}\right)$. Tensile or Young modulus, shear modulus and bulk modulus are used to indicate the strength of a rigid material under different stress conditions, namely the ability to resist elastic deformation. Elastic and plastic properties of materials, such as hardness, tensile strength, rupture strength and the tensile length, can be associated with elastic modulus. Hardness and tensile strength represents the size of the plastic deformation resistance capacity, is proportional to the shear modulus. Fracture strength is proportional to the bulk modulus 9

Table-2 shows the modulus relationship among three kinds of systems is $\mathrm{M}_{\mathrm{PVP}} \bullet \mathrm{M}_{\mathrm{PVP} / \mathrm{PVA}} \bullet \mathrm{M}_{\mathrm{PVA}}$, which has to do with

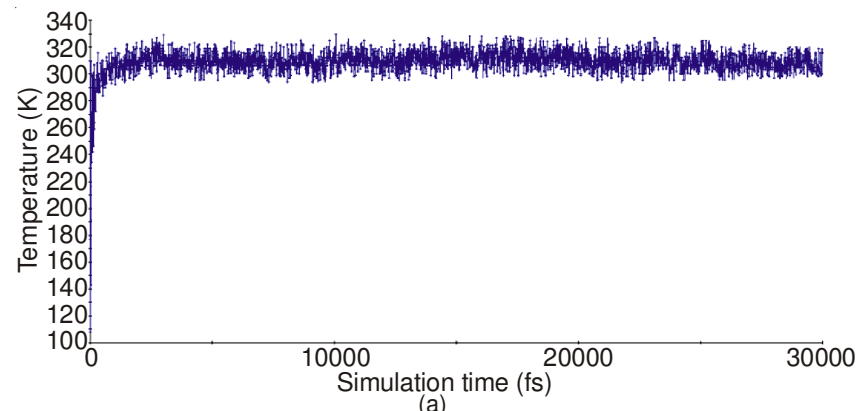

(a)

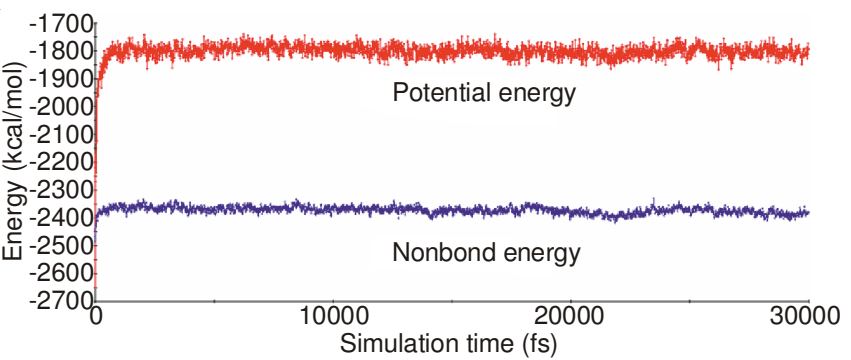

(b)

Fig. 3. Two criteria of simualtion the whole simualtion system reach equilibrium: (a) temperature $v s$. simulation time and (b) energy $v s$. simulation time

TABLE-2

ELASTIC CONSTANTS AND MECHANICAL PROPERTIES OF PVP, PVA AND PVP/PVA AT $310 \mathrm{~K}$ (Gpa)

\begin{tabular}{cccc}
\hline Elastic coefficient & PVP & PVA & PVP/PVA \\
\hline $\mathrm{C}_{11}$ & $6.19 \pm 0.30$ & $11.06 \pm 0.43$ & $10.38 \pm 0.35$ \\
$\mathrm{C}_{22}$ & $5.15 \pm 0.34$ & $11.49 \pm 0.50$ & $10.69 \pm 0.34$ \\
$\mathrm{C}_{33}$ & $11.12 \pm 0.43$ & $9.68 \pm 0.46$ & $9.02 \pm 0.62$ \\
$\mathrm{C}_{44}$ & $1.12 \pm 0.34$ & $2.52 \pm 0.38$ & $3.23 \pm 0.343$ \\
$\mathrm{C}_{55}$ & $2.99 \pm 0.26$ & $3.66 \pm 0.30$ & $2.62 \pm 0.29$ \\
$\mathrm{C}_{66}$ & $2.29 \pm 0.30$ & $2.49 \pm 0.22$ & $2.94 \pm 0.45$ \\
$\mathrm{C}_{12}$ & $-3.10 \pm 0.12$ & $5.50 \pm 0.32$ & $5.30 \pm 0.28$ \\
$\mathrm{C}_{13}$ & $-1.64 \pm 0.33$ & $4.93 \pm 0.16$ & $4.53 \pm 0.18$ \\
$\mathrm{C}_{23}$ & $0.40 \pm 0.12$ & $4.63 \pm 0.33$ & $4.21 \pm 0.33$ \\
$\mathrm{C}_{15}$ & $-2.21 \pm 0.35$ & $0.71 \pm 0.21$ & $-1.06 \pm 0.21$ \\
$\mathrm{C}_{25}$ & $-1.13 \pm 0.25$ & $0.91 \pm 0.29$ & $-0.52 \pm 0.14$ \\
$\mathrm{C}_{35}$ & $0.36 \pm 0.20$ & $-0.03 \pm 0.25$ & $-0.68 \pm 0.27$ \\
$\mathrm{C}_{46}$ & $-0.29 \pm 0.11$ & $-0.14 \pm 0.13$ & $-0.09 \pm 0.10$ \\
\hline Tensile modulus $\mathrm{E}$ & 4.31 & 7.82 & 7.06 \\
Poisson ratio & 0.33 & 0.30 & 0.32 \\
Bulk modulus & 5.38 & 6.75 & 6.46 \\
Shear modulus & 1.58 & 2.99 & 2.68 \\
$\mathrm{C}_{12}$ - $\mathrm{C}_{44}$ & -1.23 & 2.18 & 2.07 \\
\hline
\end{tabular}

weak rigidity of PVP hydrogel and consistent with the fact that its capability to resist a variety of elastic deformation is poor. The modulus of PVA is maximum, which indicates that its mechanical properties are stronger than other hydrogels. If it is combined with PVP to form a new mixed system can increase the mechanical properties of PVP significantly, thus the scope of hydrogel material can be get broaden according to the real application requirements. Meanwhile, based on the 
Cauchy stress value of three systems, the hydrogel deformation performance can be investigated after molecular dynamics simulation. In this project, the Cauchy pressure value of PVP is negative, which indicates PVP is brittle, poor ductility, prone to tensile deformation, but adding a better ductility PVA to PVP can be very good to increase the ductility of PVP hydrogel material. Table-3 lists the elastic coefficient and isotropic elastic properties calculated by molecular dynamics under the ensemble NVT at 260, 310, 360 and $410 \mathrm{~K}$.

Table-3 indicates that there is no significant change in the elasticity coefficient and modulus of PVP/PVA with temperature rising from 260 to $410 \mathrm{~K}$. It means that the mechanical properties of PVP/PVA are not affected by temperature, so the mechanical properties of PVP/PVA composite material almost do not consider the thermal factor. Poisson's ratio is the ratio of lateral strain and longitudinal strain. Table- 3 shows that the Poisson's ratio of the composite material is always 0.32 , which means that there's almost no change with temperature. Poisson's ratio of regular plastic is between 0.2 and 0.4 and the Poisson's ratio of PVP/PVA is within this range. So the mixed system has certain properties of plastics. The radial distribution function $\mathrm{g}(\mathrm{r})$ is a reflection of the physical characteristics of material microstructure, which indicates a probability density to another molecule at a distance $r$ around a place where the ratio of molecules randomly distributed with respect to the probability density. The radial distribution function was adopted by the study of the equilibrium path, which shows the interaction mechanism and nature of PVP/PVA hydrogel.

\begin{tabular}{|c|c|c|c|c|}
\hline \multicolumn{5}{|c|}{$\begin{array}{c}\text { TABLE-3 } \\
\text { MECHANICAL PROPERTIES PARAMETERS OF PVP/PVA } \\
\text { HYDROGEL AT DIFFERENT TEMPERATURES (Gpa) }\end{array}$} \\
\hline Elastic coefficient & $260 \mathrm{~K}$ & $310 \mathrm{~K}$ & $360 \mathrm{~K}$ & $410 \mathrm{~K}$ \\
\hline $\mathrm{C}_{11}$ & 10.37 & 10.38 & 10.39 & 10.39 \\
\hline $\mathrm{C}_{22}$ & 10.68 & 10.69 & 10.69 & 10.69 \\
\hline $\mathrm{C}_{33}$ & 9.00 & 9.02 & 9.02 & 9.03 \\
\hline $\mathrm{C}_{44}$ & 3.23 & 3.23 & 3.23 & 3.23 \\
\hline $\mathrm{C}_{55}$ & 2.61 & 2.62 & 2.62 & 2.63 \\
\hline $\mathrm{C}_{66}$ & 2.93 & 2.94 & 2.95 & 2.95 \\
\hline $\mathrm{C}_{12}$ & 5.29 & 5.30 & 5.31 & 5.31 \\
\hline $\mathrm{C}_{13}$ & 4.52 & 4.53 & 4.53 & 4.52 \\
\hline $\mathrm{C}_{23}$ & 4.19 & 4.21 & 4.21 & 4.21 \\
\hline $\mathrm{C}_{15}$ & -1.07 & -1.06 & -1.08 & -1.09 \\
\hline $\mathrm{C}_{25}$ & -0.51 & -0.52 & -0.52 & -0.53 \\
\hline $\mathrm{C}_{35}$ & -0.67 & -0.68 & -0.69 & -0.69 \\
\hline $\mathrm{C}_{46}$ & -0.09 & -0.09 & -0.10 & -0.11 \\
\hline Tensile modulus E & 7.05 & 7.06 & 7.06 & 7.07 \\
\hline Poisson ratio & 0.32 & 0.32 & 0.32 & 0.32 \\
\hline Bulk modulus & 6.45 & 6.46 & 6.46 & 6.47 \\
\hline Shear modulus & 2.68 & 2.68 & 2.68 & 2.68 \\
\hline $\mathrm{C}_{12}-\mathrm{C}_{44}$ & 2.06 & 2.07 & 2.08 & 2.08 \\
\hline
\end{tabular}

Poly(vinyl alcohol) chain contains carbon atom of backboned, oxygen and hydrogen atom of hydroxy and the hydrogen atom associated to backbone carbon atom. Carbon atom is surrounded by hydrogen atom and methyl group. Therefore, it is hardly to react with PVP, then the only three atoms need to be taken into account: $\mathrm{O}_{\mathrm{a}}, \mathrm{H}_{\mathrm{a} 1}$ and $\mathrm{H}_{\mathrm{a} 2}$. Similarly, PVP chain contains carbon atom, hydrogen atom, oxygen atom and nitrogen atom. And among these atoms, carbon atom is surrounded by hydrogen atom and methyl group, nitrogen atom is surrounded by three carbon atoms in like manner. Thus only hydrogen atom and oxygen have been left and they are credited as $\mathrm{H}_{\mathrm{b}}$ and $\mathrm{O}_{\mathrm{b}}$. The graph of $\mathrm{g}(\mathrm{r})-\mathrm{r}$ relationship is listed as follows. The radial distribution function of atoms can show interaction pattern and principle between non-bonded atoms. In general, the peak value lower than $3.5 \AA$ is mainly composed of chemical bond and hydrogen bond and the value between 3.5-5 $\AA$ is mainly conducted by van der Waals (vdW) result.
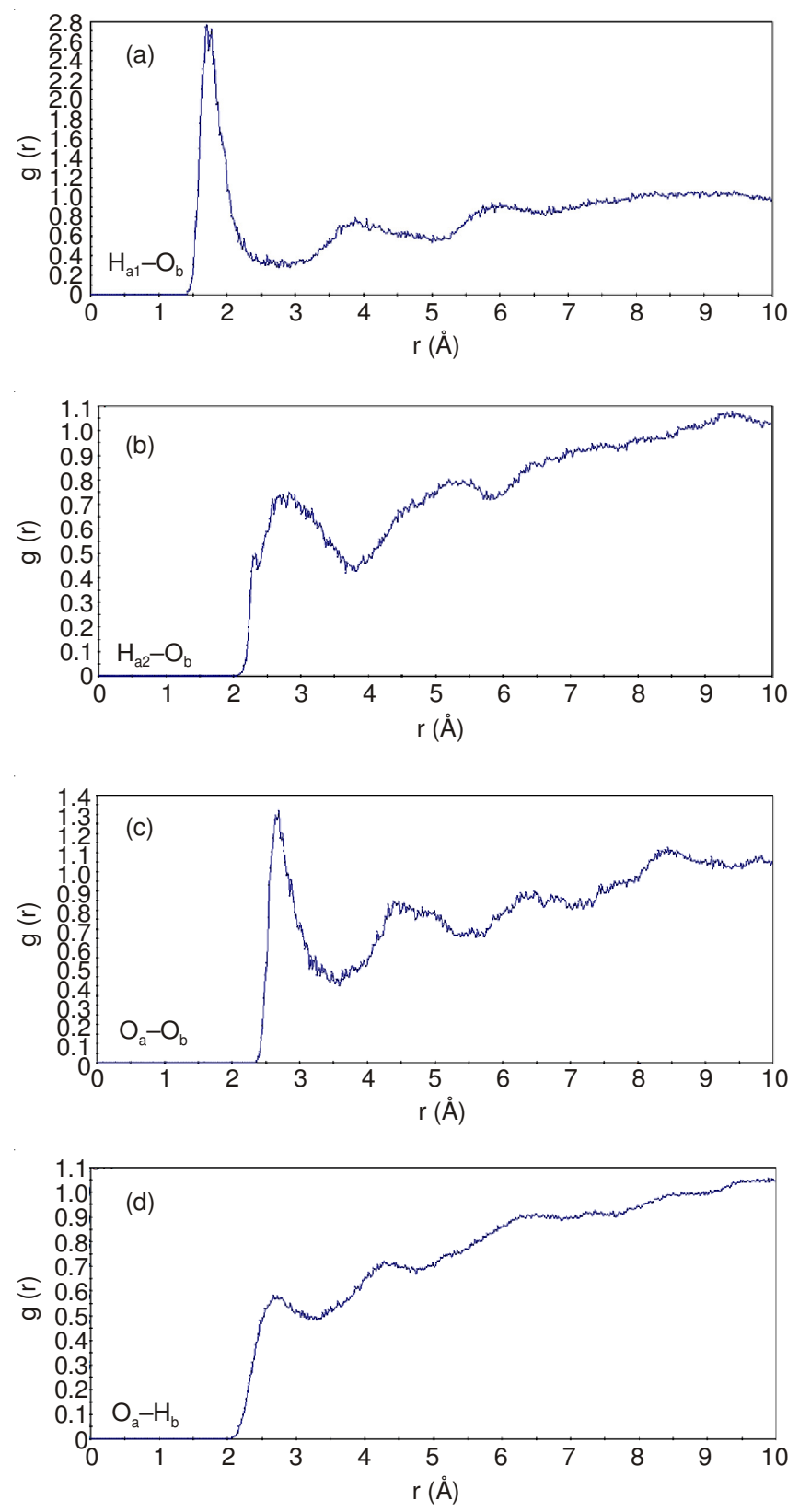

Fig. 4. Radial distribution function of PVP/PVA hydrogel

Fig. 4(a) indicated, the value reaches the peak nearby $r=$ $1.8 \AA$, which is slightly bigger than covalent radius of hydrogen and oxygen. This shows that $\mathrm{O}$ of PVP and $\mathrm{H}$ of PVA form the hydrogen bond; the $\mathrm{H}_{\mathrm{a} 2}-\mathrm{O}_{\mathrm{b}}$ function in Fig. 4(b) run up to the first peak value close to $r=2.8 \AA$ and reach the highest value while $\mathrm{r}>3.5 \AA$, this means that $\mathrm{H}_{\mathrm{a} 2}$ atom and $\mathrm{O}_{\mathrm{b}}$ atom is mainly carried by van der Waals result, while a few amount form the hydrogen bond; the function of $\mathrm{O}_{\mathrm{a}}-\mathrm{O}_{\mathrm{b}}$ in Fig. 4(c) reach the 
first peak at the nearby $r=2.7 \AA$, which is mainly caused by the hydrogen bond between $\mathrm{H}_{\mathrm{a} 1}$ and $\mathrm{O}_{\mathrm{b}}$, reducing the distance among $\mathrm{O}_{\mathrm{a} 1}$ and $\mathrm{O}_{\mathrm{b}}$; The radial distribution in Fig. 4(d) is similar as Fig. 4(b). Obviously, van der Waals forces are the dominant interactions between $\mathrm{O}_{\mathrm{a}}$ of PVP and $\mathrm{H}_{\mathrm{b}}$ of PVA. The study of the radial distribution function indicated: the interactions between PVA and PVP are expected to occur through interchain hydrogen bonding between the oxygen of PVP and the hydrogen of PVA, which shows the fact that the oxygen of PVP is easy to combine with the hydrogen of PVA by hydrogen bond.

\section{Conclusion}

Compared with pure PVP material, PVP/PVA composite material has a better modulus and favourable behaviour of mechanical properties, thus broadening the application of this material. The ductility of pure PVP can be improved through combing with PVA into PVP/PVA hybrid system. There is no significant change in elasticity coefficient and modulus of PVP/ PVP within the temperature range of 260-410 K, which shows that mechanical properties of this material are not affected by the temperature fluctuation. The interaction between PVA and PVP is expected to occur through interchain hydrogen bonding between the oxygen of PVP and the hydrogen of PVA, which shows the fact that the oxygen of PVP is cushy for combination with the hydroxyl of PVA through hydrogen bond.

\section{ACKNOWLEDGEMENTS}

This project is sponsored by the Natural Science Foundation of China (No.51175432), the Fundamental Research Funds for the Central Universities (3102014JCS05007), the Doctor Special Science and Technological Funding of the China Ministry of Education (Grant No. 20116102110046), and the Graduate Starting Seed Fund of Northwestern Polytechnical University (No. Z2014037).

\section{REFERENCES}

1. Z.H. Lin, W.H. Wu, J.Q. Wang and X. Jin, React. Funct. Polym., 67, 789 (2007).

2. S.M. Li, Z. Xiu and J.Y. Xin, J. Tech. Dev. Chem. Ind., 39, 13 (2010).

3. Z.-M. Chen, J.-Q. Xiong, D.-G. Liao and H. Li, J. Funct. Mater, 43, 457 (2012).

4. J.-J. Zhang, Y.-C. Meng, X.-F. Chen, Y.-L. Li, X.-G. Luo and C. Lin, J. Med. Biomech., 25, 16 (2010).

5. B. Prathab, V. Subramanian and T.M. Aminabhavi, Polymer, 48, 409 (2007).

6. L. Xia, J.-J. Xiao, J.-F. Fan, W. Zhu and H.-M. Xiao, Acta Chim. Sin., 66, 874 (2008).

7. R.J. Swenson, Am. J. Phys., 51, 940 (1983).

8. J.P. Watt, G.F. Davies and R.J. O'Connell, J. Space Phys., 14, 541 (1976).

9. S.F. Pugh, Philos. Mag. A, 45, 823 (1954). 